BNL-HET-98/38

hep-ph/9812394

\title{
The muon anomalous magnetic moment in QED: three-loop electron and tau contributions
}

\author{
Andrzej Czarnecki \\ Physics Department, Brookhaven National Laboratory, \\ Upton, NY 11973, USA \\ Maciej Skrzypek \\ Institute of Nuclear Physics, ul. Kawiory 26a, 30-055 Cracow, Poland \\ and \\ CERN, Theory Division, CH-1211 Geneva 23, Switzerland
}

\begin{abstract}
We present an analytic calculation of electron and tau $\mathcal{O}\left(\alpha^{3}\right)$ loop effects on the muon anomalous magnetic moment. Computation of such three-loop diagrams with three mass scales is possible using asymptotic and eikonal expansions. An evaluation of a new type of eikonal integrals is presented in some detail.
\end{abstract}




\section{Introduction}

The new measurement of the muon anomalous magnetic moment, $a_{\mu}=\left(g_{\mu}-2\right) / 2$, in the experiment E821 in Brookhaven has motivated many recent theoretical studies. In the Standard Model, $a_{\mu}$ receives contributions from electromagnetic and weak interactions, as well as from loop effects involving hardrons. All three types of effects have been studied recently. QED contributions are known to the four-loop level, and even some five-loop diagrams have been evaluated. The next largest contribution is due to hadronic loops and is the most difficult one to evaluate. There has been recently significant progress in both evaluation of the light-by-light diagrams [1] and hadronic vacuum polarization effects [2]. Electroweak two-loop effects are also known [3].

The present paper is devoted to the only 3-loop QED contribution to $a_{\mu}$ that has not been evaluated analytically so far: from a diagram with electron and $\tau$ lepton loop insertions in the photon propagator, shown in Fig. 1. Because of the three

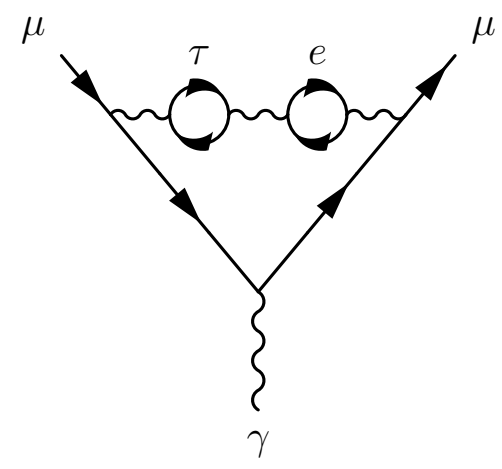

Figure 1: Three-loop contribution to muon anomalous magnetic moment with the electron and tau loops inserted in the photon propagator.

mass scales present in this diagram, $m_{e, \mu, \tau}$, it cannot at present be evaluated in a closed form. However, we present an approach based on asymptotic and eikonal expansions, which takes advantage of the wide separations between those scales and permits an evaluation with an arbitrary accuracy.

We begin with a brief summary of the present knowledge of QED contributions to the electron and muon anomalous magnetic moments, $a_{e, \mu}$ (for more details see e.g. [4]).

(a) Electron. To match the present experimental precision, one needs four terms in the expansion of $a_{e}$ in the fine structure constant $\alpha$,

$$
a_{e}=\sum_{n=1}^{4} A_{n}\left(\frac{\alpha}{\pi}\right)^{n}+\ldots
$$

where ellipses indicate contributions of loops containing the heavy leptons $\mu$ and $\tau$; $A_{1,2}$ have been known since the early years of QED [5]. An analytical evaluation 
of $A_{3}$ required the efforts of many groups and took almost 40 years; it has been completed only recently [6]. $A_{4}$ is known only numerically.

The perturbative series for $a_{e}$ is very well behaved, and, together with the most recent experimental values for electron and positron [7], allows one to deduce a very precise value of the fine structure constant $[8,4]$,

$$
\alpha^{-1}=137.03599959(38)(13) \text {. }
$$

(b) Muon. The value of $\alpha$ found from electron $g-2$, eq. (2), can be applied to compute the QED contribution to the muon anomalous magnetic moment $a_{\mu}$. Because of the presence of electron loops, higher-order QED contributions to $a_{\mu}$ are enhanced with respect to $a_{e}$. At present five terms of the expansion in $\alpha$ are needed:

$$
a_{\mu}^{\mathrm{QED}}=\sum_{n=1}^{5} C_{n}\left(\frac{\alpha}{\pi}\right)^{n}
$$

with

$$
\begin{aligned}
C_{1} & =A_{1}=0.5, \\
C_{2} & =A_{2}+a_{1}\left(m_{e} / m_{\mu}\right)+a_{2}\left(m_{\mu} / m_{\tau}\right)=0.765857388(44), \\
C_{3} & =A_{3}+C_{3}^{\gamma \gamma}(e)+C_{3}^{\gamma \gamma}(\tau)+C_{3}^{\text {vac. pol. }}(e)+C_{3}^{\text {vac. pol. }}(\tau)+C_{3}^{\text {vac. pol. }}(e, \tau) \\
& =24.050509(2), \\
C_{4} & =A_{4}+127.55(41)=126.04(41), \\
C_{5} & =930(170),
\end{aligned}
$$

where $a_{1,2}$ describe contributions of two-loop diagrams with electron and tau loops, respectively $[9,10]$. We will discuss them in detail later on.

In $C_{3}$ we have contributions from light-by-light scattering diagrams with $e$ and $\tau$ loops [11], and vacuum polarization diagrams with either $e$, or $\tau$ [12], or both types of loops. An analytical evaluation of this latter contribution of mixed $e-\tau$ diagram, $C_{3}^{\text {vac. pol. }}(e, \tau)$, is the main purpose of this paper and will be presented below. Numerically it can be evaluated using the kernel from [13]:

$$
C_{3}^{\text {vac. pol. }}(e, \tau)=0.0005276(2)
$$

For $C_{4}$ one uses the difference between the muon and electron coefficients found in [14]. For $C_{5}$ only a numerical estimate of the presumably dominant contributions is known [15].

The present estimate of the total QED contribution to $a_{\mu}$ is [4]

$$
a_{\mu}^{\mathrm{QED}}=116584705.6(2.9) \times 10^{-11} .
$$




\section{Two-loop diagrams with electron and tau loops}

Before calculating the contribution of the three-loop diagram of Fig. 1, we would like to discuss a method of evaluating the two-loop diagrams shown if Fig. 2 (we discuss only the Pauli formfactor, relevant for $a_{\mu}$ ). It will serve us as an example to illustrate the main points of our calculational techniques. Of course, full analytical

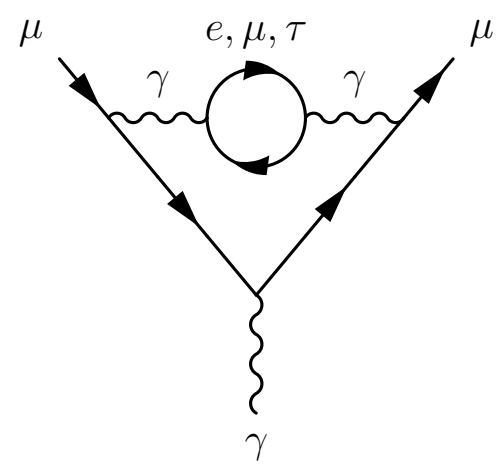

Figure 2: Two-loop contributions of lepton loops in the photon propagator.

results for these two-loop contributions are known $[9,10]$. It is instructive to note how they were obtained. First, a closed analytical formula was found, with full functional dependence on the ratio of masses $m_{e} / m_{\mu}$ or $m_{\mu} / m_{\tau}$ [10]. That formula, containing dilogarithms, was found awkward to use, because of cancellations and difficulties in estimating the accuracy in the numerical evaluation. In [9] expansions of the exact result in powers of small mass ratios were given. Such expansions avoid evaluation of special functions and their accuracy can be precisely assessed.

Here we demonstrate how such expansions can be obtained without knowledge of the exact result. We first consider the case of the $\tau$ loop insertion. In this case a well known method of heavy mass expansion is applicable (for a review see [16]). Let us denote the loop momenta by $p_{\tau}$ and $p_{\mu}$, for the momentum flowing inside the $\tau$ loop and for that in the virtual photon respectively. There are two regions of integration, with characteristic scales of momenta $p_{\tau} \sim p_{\mu} \sim m_{\tau}$ and $p_{\tau} \sim m_{\tau}, p_{\mu} \sim m_{\mu}$. In the case of the first region, which we can call the hard contribution, we can safely regard the muon mass and external momentum as small with respect to the integration momenta, and expand the integrand in these small parameters. The resulting integral corresponds to a vacuum diagram shown in Fig. 3(a). In the second region we cannot neglect the external muon momentum, but now, since $p_{\mu} \ll p_{\tau}$, we can expand the $\tau$ loop propagators in Taylor series in $p_{\mu}$, so that the integral factorizes into a product of two one-loop diagrams, shown in Fig. 3(b). This nice factorization of the relevant integration regions is only possible in regularization schemes, which do not introduce additional mass scales, such as dimensional regularization.

After evaluation of these two sub-diagrams and renormalization of the electric charge of the muon, we find a finite result. Several of the terms we calculated agree with 


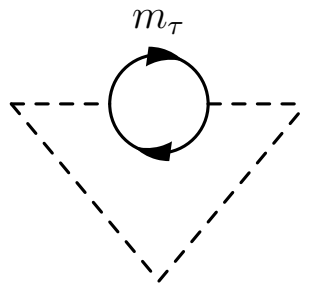

(a)

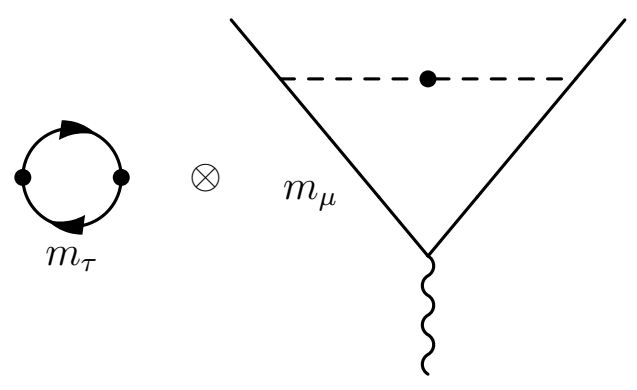

(b)

Figure 3: Graphic representation of characteristic momentum scales in the two-loop diagram with a $\tau$ loop. Solid and dashed lines denote, respectively, massive and massless propagators.

formula (12) in [9]:

$$
\begin{aligned}
a_{2}(l= & \left.m_{\mu} / m_{\tau}\right)=\frac{l^{2}}{45}+\frac{l^{4} \ln l}{70}+\frac{9}{19600} l^{4}-\frac{131}{99225} l^{6}+\frac{4 l^{6}}{315} \ln l \\
& -\sum_{n=3}^{\infty} \frac{\left(8 n^{3}+28 n^{2}-45\right) l^{2 n+2}}{[(n+3)(2 n+3)(2 n+5)]^{2}}+2 \ln l \sum_{n=3}^{\infty} \frac{n l^{2 n+2}}{(n+3)(2 n+3)(2 n+5)} .
\end{aligned}
$$

The other two-loop diagram that we have to consider is the electron loop insertion in the photon propagator (Fig. 2). Here the situation is somewhat more involved, since there are now three integration regions. Introducing the obvious notation for the integration momenta, $p_{e, \mu}$, we have: $\left(p_{e} \sim p_{\mu} \sim m_{\mu}\right),\left(p_{e} \sim m_{e}\right.$ and $\left.p_{\mu} \sim m_{\mu}\right)$, and $\left(p_{e} \sim p_{\mu} \sim m_{e}\right)$. The first two regions correspond to known cases of the "large momentum expansion" [16]. They are depicted in Fig. 4(a,b). Figure 4(a) denotes a simple Taylor expansion of the electron propagators in $m_{e}$, justified if both integration momenta are large. In Fig. 4(b) we have illustrated one of the two cases, where one of the electron lines cannot be expanded in $m_{e}$, but where the integration factorizes and we have a product of one-loop diagrams. More exotic is the third case, Fig. 4(c), where the only scale of integration is $m_{e}$, since all dependence on the muon mass and external momentum factorizes. Sub-diagrams of this type have been encountered in a different context in eikonal expansions $[17,18]$. Integrals that arise in the present case are somewhat different and we give here some details of their evaluation.

Since now the integration momentum $p_{\mu}$ is much smaller than the external muon momentum $p$, we can expand muon propagators in $p_{\mu}^{2}$. As a result we have to compute integrals of the form

$$
J\left(a_{1}, a_{2}\right)=\frac{1}{\pi^{D}} \int \frac{\mathrm{d}^{D} p_{\mu} \mathrm{d}^{D} p_{e}}{\left(p_{\mu}^{2}\right)^{a_{1}}\left(2 p_{\mu} \cdot p\right)^{a_{2}}\left(p_{e}^{2}+m_{e}^{2}\right)\left[\left(p_{\mu}+p_{e}\right)^{2}+m_{e}^{2}\right]} .
$$




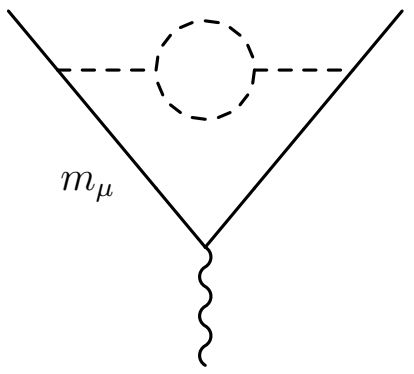

(a)

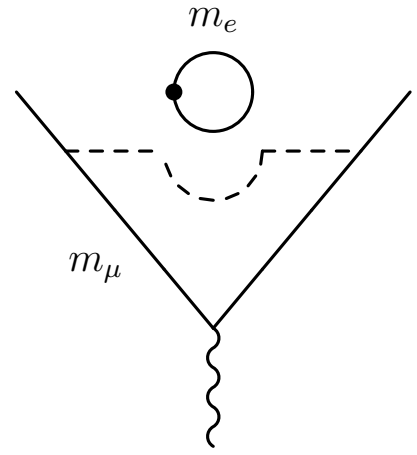

(b)

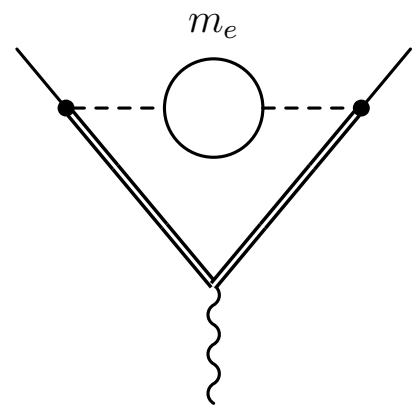

(c)

Figure 4: Integration regions contributing to the two-loop diagram with an electron loop. (a): Taylor expansion in $m_{e}$. (b): region of soft electron loop momentum. (c): region of both momenta soft (double line denotes muon propagator expanded in the square of its virtual momentum).

First, we use Feynman parameters to combine the last two propagators and integrate over $p_{e}$. We get

$$
J\left(a_{1}, a_{2}\right)=\frac{1}{\pi^{D / 2}} \Gamma(\epsilon) x^{-\epsilon}(1-x)^{-\epsilon} \int \frac{\mathrm{d}^{D} p_{\mu}}{\left(p_{\mu}^{2}\right)^{a_{1}}\left(2 p_{\mu} \cdot p\right)^{a_{2}}\left(p_{\mu}^{2}+m_{x}^{2}\right)^{\epsilon}},
$$

with $m_{x}^{2} \equiv m_{e}^{2} / x(1-x)$. Next, using again Feynman parameters, we combine the first and the last term in the denominator. Finally, using

$$
\frac{1}{a^{\alpha} b^{\beta}}=\frac{1}{B(\alpha, \beta)} \int_{0}^{\infty} \mathrm{d} \lambda \frac{\lambda^{\beta-1}}{[a+b \lambda]^{\alpha+\beta}},
$$

and integrating over $p_{\mu}$, we get

$$
\begin{gathered}
J\left(a_{1}, a_{2}\right)=\frac{\Gamma\left(\frac{a_{2}}{2}\right) \Gamma\left(a_{1}+\frac{a_{2}}{2}-2+2 \epsilon\right) \Gamma\left(2-a_{1}-\frac{a_{2}}{2}-\epsilon\right) \Gamma^{2}\left(-1+a_{1}+\frac{a_{2}}{2}+\epsilon\right)}{2 \Gamma\left(a_{2}\right) \Gamma\left(2-\frac{a_{2}}{2}-\epsilon\right) \Gamma\left(-2+2 a_{1}+a_{2}+2 \epsilon\right)} \\
\times \frac{m_{e}^{4-2 a_{1}-a_{2}-4 \epsilon}}{m_{\mu}^{a_{2}}} \\
=\frac{-\pi^{2} \Gamma\left(a_{1}+\frac{a_{2}}{2}-2+2 \epsilon\right) m_{e}^{4-2 a_{1}-a_{2}-4 \epsilon}}{2^{2 a_{1}+2 a_{2}-3+2 \epsilon} \Gamma\left(\frac{a_{2}+1}{2}\right) \Gamma\left(a_{1}+\frac{a_{2}-1}{2}+\epsilon\right) \Gamma\left(2-\frac{a_{2}}{2}-\epsilon\right) \sin \pi\left(a_{1}+\frac{a_{2}}{2}+\epsilon\right) m_{\mu}^{a_{2}}} .
\end{gathered}
$$

We should mention that the integrand in eq. (7) could also contain products $p \cdot p_{e}$. It is possible to replace them by combinations of products of $p_{\mu} \cdot p_{e}$ and $p_{\mu} \cdot p$ using traceless products (see e.g. [17]). However, in the present case there are at most two powers of $p_{e}$ in the numerator and we can use the following simple formulas:

$$
\left(p \cdot p_{e}\right)^{2} \rightarrow \frac{p^{2} p_{e}^{2}}{D}+\left[\left(p_{\mu} \cdot p\right)^{2}-\frac{p^{2} p_{\mu}^{2}}{D}\right] \frac{D\left(p_{e} \cdot p_{\mu}\right)^{2}-p_{e}^{2} p_{\mu}^{2}}{(D-1)\left(p_{\mu}^{2}\right)^{2}}, \quad p \cdot p_{e} \rightarrow \frac{\left(p \cdot p_{\mu}\right)\left(p_{\mu} \cdot p_{e}\right)}{p_{\mu}^{2}} .
$$


Adding the contributions of the three integration regions, we find, after renormalization, that the terms we obtained agree with formula (11) of [9]:

$$
\begin{aligned}
a_{1}\left(k=m_{e} / m_{\mu}\right)= & -\frac{25}{36}+\frac{\pi^{2}}{4} k-\frac{1}{3} \ln k+(3+4 \ln k) k^{2}-\frac{5}{4} \pi^{2} k^{3} \\
& +\left[\frac{\pi^{2}}{3}+\frac{44}{9}-\frac{14}{3} \ln k+2 \ln ^{2} k\right] k^{4}+\frac{8}{15} k^{6} \ln k-\frac{109}{225} k^{6} \\
& +\sum_{n=2}^{\infty}\left[\frac{2(n+3)}{n(2 n+1)(2 n+3)} \ln k-\frac{8 n^{3}+44 n^{2}+48 n+9}{n^{2}(2 n+1)^{2}(2 n+3)^{2}}\right] k^{2 n+4} .
\end{aligned}
$$

\section{Three-loop diagram with $e$ and $\tau$ loop inser- tions}

We now proceed to the actual focus of our work, the contribution of the three-loop diagram with $e$ and $\tau$ loop insertions, shown in Fig. 1. So far its contribution to $a_{\mu}$ has been evaluated only numerically [12].

Using the techniques described above, we can easily obtain an expansion of the Pauli part of this diagram with arbitrary accuracy. There are now three integration momenta and we have to consider five integration regions, combinations of the con-

ditions described in the context of two-loop diagrams. Using the notation $p_{\tau, \mu, e}$ for the integration momenta in the three loops, the regions we have to consider are:

- $p_{\tau} \sim p_{\mu} \sim p_{e} \sim m_{\tau}$,

- $p_{\tau} \sim m_{\tau} ; p_{\mu} \sim p_{e} \sim m_{\mu}$

- $p_{\tau} \sim m_{\tau} ; p_{\mu} \sim m_{\mu} ; p_{e} \sim m_{e}$

- $p_{\tau} \sim m_{\tau} ; p_{\mu} \sim p_{e} \sim m_{e}$

- $p_{\tau} \sim p_{\mu} \sim m_{\tau} ; p_{e} \sim m_{e}$.

Calculations in each of these regions are analogous to the cases described in the previous section. For present purposes it is more than sufficient to retain the first three terms in the $m_{\mu}^{2} / m_{\tau}^{2}$ expansion and two terms in $m_{e}^{2} / m_{\tau}^{2}$. After renormalization we find

$$
\begin{aligned}
& C_{3}^{\text {vac. pol. }}(e, \tau) \simeq \frac{m_{\mu}^{2}}{m_{\tau}^{2}}\left(\frac{4}{135} \ln \frac{m_{\mu}}{m_{e}}-\frac{1}{135}\right) \\
& +\frac{m_{\mu}^{4}}{m_{\tau}^{4}}\left(-\frac{229213}{12348000}+\frac{\pi^{2}}{630}-\frac{37}{11025} \ln \frac{m_{\tau}}{m_{\mu}}-\frac{1}{105} \ln \frac{m_{\tau}}{m_{\mu}} \ln \frac{m_{\tau} m_{\mu}}{m_{e}^{2}}+\frac{3}{4900} \ln \frac{m_{\mu}}{m_{e}}\right)
\end{aligned}
$$




$$
\begin{aligned}
+\frac{m_{\mu}^{6}}{m_{\tau}^{6}} & \left(-\frac{1102961}{75014100}+\frac{4 \pi^{2}}{2835}-\frac{398}{297675} \ln \frac{m_{\tau}}{m_{\mu}}-\frac{8}{945} \ln \frac{m_{\tau}}{m_{\mu}} \ln \frac{m_{\tau} m_{\mu}}{m_{e}^{2}}-\frac{524}{297675} \ln \frac{m_{\mu}}{m_{e}}\right) \\
& +\frac{2}{15} \frac{m_{e}^{2}}{m_{\tau}^{2}}-\frac{4 \pi^{2}}{45} \frac{m_{e}^{3}}{m_{\tau}^{2} m_{\mu}} \\
= & 0.0005276(2),
\end{aligned}
$$

in agreement with the numerical evaluation. The error in the result is due to the $\tau-$ lepton mass uncertainty. The leading-logarithmic term of this expansion corresponds to simply replacing $\alpha\left(q^{2}=0\right)$ by $\alpha\left(m_{\mu}^{2}\right)$ in the two-loop diagram with a $\tau$ loop. We have included the last term, with odd powers of $m_{e}$ and $m_{\mu}$, even though it is not relevant numerically. It illustrates typical contributions of the eikonal expansion, the only source of terms non-analytical in masses squared.

We have checked eq. (10) by comparing it with an analytical integration, using the kernel function given in [13]. Terms quadratic in masses $m_{\tau, \mu, e}$ are in complete agreement. The odd powers of $m_{e}$ have not been included in [13].

With formula (10) the complete QED contribution to $a_{\mu}$ is now known analytically. In this particular case this result does not noticeably improve the accuracy of the QED prediction, since the error in (10) comes from the $\tau$ lepton mass measurement. However, the technique presented here might facilitate other calculations. We have seen that a combination of large momentum, heavy mass, and eikonal expansions eliminates the need for numerical calculations and enables us to construct arbitrarily accurate expansions without knowledge of the exact result.

\section{Acknowledgements}

We thank the CERN Theory Group for support and hospitality during our visit, where most of this project was completed. This work was supported in part by the U.S. Department of Energy under grant number DE-AC02-98CH10886, Polish Government grants numbers KBN 2P03B08414, KBN 2P03B14715, and the Maria Skłodowska-Curie Joint Fund II PAA/DOE-97-316.

\section{References}

[1] J. Bijnens, E. Pallante and J. Prades, Nucl. Phys. B474, 379 (1996).

M. Hayakawa and T. Kinoshita, Phys. Rev. D57, 465 (1998).

[2] S. Eidelman and F. Jegerlehner, Z. Phys. C67, 585 (1995).

M. Davier and A. Höcker, hep-ph/9805470.

[3] A. Czarnecki, B. Krause and W. Marciano, Phys. Rev. Lett. 76, 3267 (1996). 
[4] A. Czarnecki and W. J. Marciano, hep-ph/9810512, talk given at 5th International Workshop on Tau Lepton Physics, Santander, Spain, September 1998.

[5] J. Schwinger, Phys. Rev. 73, 416 (1948).

C. M. Sommerfield, Phys. Rev. 107, 328 (1957); Ann. Phys. 5, 26 (1958).

A. Petermann, Nucl. Phys. 3, 689 (1957); Helv. Phys. Acta 30, 407 (1957).

[6] S. Laporta and E. Remiddi, Phys. Lett. B379, 283 (1996).

[7] R. S. van Dyck Jr., P. B. Schwinberg and H. G. Dehmelt, Phys. Rev. Lett. 59, 26 (1987).

[8] T. Kinoshita, hep-ph/9808351, talk given at International Workshop on Hadronic Atoms and Positronium in the Standard Model, Dubna, Russia, May 1998.

[9] G. Li, R. Mendel and M. A. Samuel, Phys. Rev. D47, 1723 (1993).

[10] H. H. Elend, Phys. Lett. 20, 682 (1966); erratum: ibid., 21, 720.

[11] S. Laporta and E. Remiddi, Phys. Lett. B301, 440 (1993).

[12] S. Laporta, Nuovo Cim. 106A, 675 (1993).

[13] B. Krause, Phys. Lett. B390, 392 (1997).

[14] T. Kinoshita, Phys. Rev. D47, 5013 (1993).

[15] S. G. Karshenboim, Phys. Atom. Nucl. 56, 857 (1993).

[16] K. G. Chetyrkin, preprint MPI-Ph/PTh 13/91.

V. A. Smirnov, Mod. Phys. Lett. A10, 1485 (1995).

F. V. Tkachev, Sov. J. Part. Nucl. 25, 649 (1994).

[17] A. Czarnecki and V. A. Smirnov, Phys. Lett. B394, 211 (1997).

[18] V. A. Smirnov, Phys. Lett. B394, 205 (1997). 